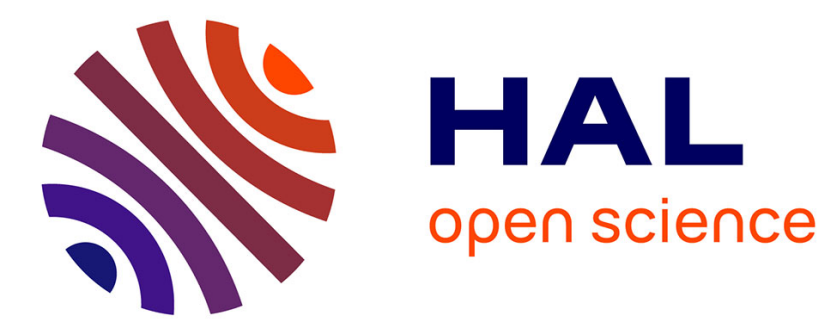

\title{
FAIRE FACE À LA DISCRIMINATION ETHNIQUE: STRATÉGIES DE DISCRIMINÉS
}

\author{
Juan Matas
}

\section{To cite this version:}

Juan Matas. FAIRE FACE À LA DISCRIMINATION ETHNIQUE: STRATÉGIES DE DISCRIM-

INÉS. Migrations Société, 2011, Migrations, racismes, résistances, 23 (133), pp.95-110. hal-01285037

\section{HAL Id: hal-01285037 \\ https://hal.science/hal-01285037}

Submitted on 8 Mar 2016

HAL is a multi-disciplinary open access archive for the deposit and dissemination of scientific research documents, whether they are published or not. The documents may come from teaching and research institutions in France or abroad, or from public or private research centers.
L'archive ouverte pluridisciplinaire HAL, est destinée au dépôt et à la diffusion de documents scientifiques de niveau recherche, publiés ou non, émanant des établissements d'enseignement et de recherche français ou étrangers, des laboratoires publics ou privés. 


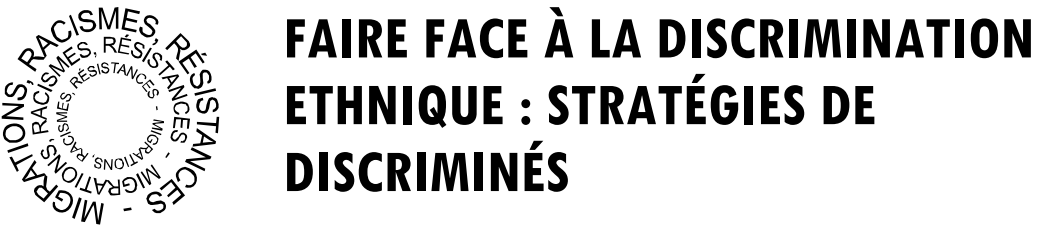

Juan MATAS *

Le phénomène de la discrimination, qui se décline de façon plurielle, est sans doute aussi ancien que les sociétés humaines elles-mêmes. Néanmoins, il se modifie au gré des mutations que ces sociétés connaissent, s'aggrave ou s'atténue, apparaît ou disparaît dans tel domaine, suit une évolution différente dans tel autre. Si la dimension historique a ici une importance majeure, les dimensions spatiale et culturelle ne sont pas moindres ; ainsi, même dans des sociétés aux caractéristiques relativement proches, la discrimination peut avoir un volume (et des contenus) tout à fait différent(s) de l'une à l'autre, pour des raisons qui s'inscrivent dans leur histoire, récente ou plus éloignée, mais aussi dans des caractéristiques culturelles et des enjeux actuels desdites sociétés.

\section{À propos de la discrimination ethnique}

Pour étudier les caractéristiques de la discrimination ethnique et les conséquences qu'elle peut avoir, il ne faut pas l'isoler mais, au contraire, la mettre en rapport avec d'autres discriminations qui ont bien des points communs avec elle. Les diverses formes de discrimination et de ségrégation renvoient toutes vers une attitude de repli, de peur, d'entre-soi, de volonté de tenir à distance l'Autre pour mieux affirmer sa supériorité sur lui.

Ce qui s'applique à la discrimination à l'égard des "minorités visibles", des handicapés ou des femmes se rejoue dans le domaine des rapports entre les classes sociales. Nous pouvons nous appuyer ici sur la remarquable analyse d'Éric Maurin, dans Le ghetto français : "I La dramaturgie française de la ségrégation urbaine n'est pas celle d'un incendie soudain et local, mais celle d'un verrovillage général, durable et silencieux des espaces

* Maître de conférences, Laboratoire Cultures et Sociétés en Europe, Faculté des sciences sociales (CNRS - Université de Strasbourg). 
et des destins sociaux. Le tableau des inégalités territoriales révèle une société extraordinairement compartimentée, où les frontières de voisinage se sont durcies et où la défiance et la tentation séparatiste s'imposent comme les principes structurants de la coexistence sociale. De fait, le "ghetto français" n'est pas tant le lieu d'un affrontement entre inclus et exclus que le théâtre sur lequel chaque groupe s'évertue à fuir ou à contourner le groupe immédiatement inférieur dans l'échelle des difficultés [...]. Les quartiers sensibles doivent demeurer naturellement un sujet de préoccupation, mais ils ne sont que le résultat le plus visible de la ségrégation urbaine. Le principe actif de la fragmentation territoriale se trouve ailleurs, dissimulé dans les plis d'une expérience infiniment plus générale mais qui reste à ce jour sans formulation politique : la réduction à tout prix de l'incertitude des rencontres et de la variété du voisinage par où se définissait un certain idéal de sociabilité urbaine [...]. Si le territoire est l'enjeu d'une compétition aussi âpre, c'est que le lieu de résidence et les interactions sociales qu'il conditionne comptent parmi les ressources essentielles d'une concurrence généralisée pour les meilleurs destins, laquelle s'engage désormais dès l'enfance. Pour comprendre la ségrégation territoriale, c'est l'importance de ces ressources qu'il faut cerner $1{ }^{1}$.

On voit ici comment se matérialise un des effets majeurs de l'extension et de la concrétisation du principe égalitaire, à savoir la recherche de nouvelles stratégies pour assurer la perpétuation de la domination de classe. C'est au sein de celle-ci que se trouve insérée la question de la discrimination ethnique, et c'est en tant que groupe d'abord vulnérable par ses caractéristiques socioéconomiques que les minorités ethniques se trouvent aux prises avec ce phénomène. Cela ne veut pas dire que la "question raciale" n'ait pas une certaine autonomie, comme nous aurons l'occasion de le voir plus loin, mais il ne faut pas pour autant la détacher de la "question sociale".

La discrimination ethnique, manifestation du racisme, est une réalité ancienne dans les sociétés humaines, même si ses formes se modifient et, globalement, les discours qui l'accompagnent et la justifient tendent à être davantage euphémisés. On a également tendance à se déculpabiliser en faisant valoir que le racisme est pratiqué non pas par ceux qui désignent l'Autre comme radicalement différent (par la couleur de sa peau, sa religion, sa culture supposée, etc.), mais précisément par cet Autre... Ainsi, les discours tenus par des "personnalités" lors des émeutes urbaines de l'automne 2005 illustrent bien cette position, comme le montrent Didier et Éric Fassin : " L'explication [des émeutes] par les discriminations raciales,

1. MAURIN, Éric, Le ghetto français. Enquête sur le séparatisme social, Paris : Éd. du Seuil - La République des idées, 2004, 95 p. (voir pp. 6-8). 
dénoncées comme le "poison de la société française", selon la formule du président de la République, et données comme clé de lecture de l'événement, apparaissait [...] comme une évidence. Cette racialisation pouvait d'ailleurs, dans le même temps, prendre une valeur opposée en inversant la logique. À propos des quartiers où les violences avaient éclaté, on entendait dire qu'ils étaient composés d'un "prolétariat de l'extérieur" et de "populations à peine françaises" qui "ne parviennent pas à s'intégrer" (Jean-Claude Casanova, membre de l'Académie des sciences morales et politiques). Le racisme servait alors à dire à la fois la cause des émeutes, désormais qualifiées de "raciales" et inscrites dans une "logique de guerre civile entre les communautés", et les pratiques des émeutiers qui "dénoncent, sous l'invocation rituelle des valeurs de la République et le culte d'une égalité de façade, leur condition de métèques", témoignant ainsi de la haine "résolument nihiliste" qu'ils vouent à la société française et à ses institutions (Nicolas Baverez, membre de la direction de l'Institut Montaigne). Le problème, ce n'était plus alors "notre" racisme, mais "leur" racisme, la racialisation de la société étant comprise comme l'effet non plus de la discrimination, mais du communautarisme $1{ }^{2}$.

Plutôt que de s'interroger sur les raisons qui conduisent des jeunes au désespoir et à des actes réprouvables, lesdites "personnalités" théorisent le "racisme anti-Blancs" des métèques avec un discours tel celui que l'on pouvait entendre dans les années 1930 pour désigner les II ennemis de la France "I, tous ces immigrés (blancs, mais frappés d'une obscure bâtardise...) qui, selon les idéologues de l'Action française, étaient un danger physique et moral pour la société française de leur époque.

Confrontés aux difficultés d'un parcours social d'autant plus aléatoire qu'il se déroule dans un contexte de crise de l'emploi et de lutte pour les places disponibles, ce qui accentue la méfiance à leur égard de la part d'une partie non négligeable de la population, les jeunes d'origine extraeuropéenne font l'expérience de la discrimination dès leur plus jeune âge, et cela contribue de manière importante à façonner leur sensibilité et leurs rapports aux autres. Cependant, il est bon de rappeler que ces jeunes-là ne constituent en rien un groupe homogène et que la diversité de leur situation et de leurs représentations de la société environnante est le fruit de très nombreux facteurs, objectifs et subjectifs. En cela, ils sont à l'image des autres membres de notre société, et notamment des autres jeunes, dont ils partagent bien plus qu'on ne le croit les aspirations et les modes de vie.

2. FASSIN, Didier ; FASSIN, Éric, "À l'ombre des émeutes", in : FASSIN, Didier ; FASSIN, Éric (sous la direction de), De la question sociale à la question raciale? Représenter la société française, Paris : Éd. La Découverte, 2006, pp. 5-16 (voir p. 6). 
Robert Castel dit fort justement à ce sujet : "I Si on prend le mot culture au sens de partage de valeurs et de modes de vie communs, on doit [...] constater une forte homogénéité entre les jeunes de banlieue et les jeunes en général. Si on a pu parler d'une "culture des cités", elle n'est pas comparable à ce que les anthropologues appellent la "culture de la pauvreté" : la production et la reproduction en vase clos de comportements, de modes de vie et d'aspirations qui sont exclusivement ceux des milieux les plus défavorisés. Les jeunes des cités partagent au contraire largement les valeurs et les aspirations de la société, en particulier le goût de consommer, l'intérêt pour l'argent et pour les signes extérieurs de richesse sur lesquels ils auraient d'ailleurs plutôt tendance à en rajouter. Pour la plupart, ils aspirent à une vie "banale" ou "normale", congruente avec les valeurs des classes moyennes: fonder une famille, avoir des enfants, une bonne situation, être conformes à ce qui est requis pour réussir dans la vie [...]. Le problème qu'affrontent ces jeunes n'est pas d'être en dehors de la société ni quant à l'espace qu'ils occupent (la cité n'est pas un ghetto), ni quant au statut qui est le leur (beaucoup d'entre eux sont des citoyens et non des étrangers). Mais ils ne sont pas non plus dedans puisqu'ils n'y occupent aucune place reconnue et beaucoup d'entre eux ne paraissent pas susceptibles de pouvoir s'en ménager une $\|^{3}$.

Ni dehors, ni dedans : c'est de cet entre-deux que naissent l'angoisse et la révolte, la souffrance aussi, exprimée dans bien des discours. Néanmoins, il faut prendre en compte l'importance du temps : comme leurs devancières, les migrations non européennes rencontrent des difficultés, de l'hostilité et semblent installées dans un provisoire qui dure. Mais il s'agit d'une impression trompeuse : leur enracinement dans la société va de l'avant, et ceux que l'on désigne comme la "seconde génération" (et, de plus en plus, la "troisième génération") diffèrent à bien des égards de leurs aînés, et d'abord parce que cette société est aussi la leur.

Dans le débat à propos des questions soulevées (certains parlent des problèmes posés) par l'intégration ou la non-intégration des populations d'origine étrangère - en fait, de celles d'origine extraeuropéenne - il est souvent question de l'ethnicisation qui proviendrait du communautarisme de ces populations, ce que conteste Hélène Bertheleu : " II ne s'agit [...] pas ici de sous-estimer le poids de la catégorisation ethnique dans les relations sociales ni dans la construction des groupes et des identités en France. Nous souhaitons plutôt pointer l'idée qu'un développement partiel de ces analyses peut nuire à une approche juste et approfondie du phénomène. Elle consisterait d'une part à ne pas réduire les rapports sociaux ethniques à la seule

3. CASTEL, Robert, La discrimination négative : citoyens ou indigènes ?, Paris : Éd. du Seuil - La République des idées, 2007, 140 p. (voir pp. 37-38). 
catégorisation ethnique observée dans les interactions sociales et, d'autre part, à ne pas considérer les appartenances ethniques, lorsqu'elles sont affirmées par les minoritaires, comme une simple réaction aux catégorisations dominantes 114 . Affirmant dans sa conclusion que l'ethnicité est un rapport social universel et donc "I inséparable de cette humanisation (pour ne pas dire ethnicisation) des individus et des groupes, qui s'épanouit toujours au sein d'une culture particulière, quel que soit son statut $1{ }^{5}$, l'auteur nous appelle à la rigueur dans l'usage d'un terme quelque peu galvaudé par l'utilisation qui en est faite ainsi qu'à une approche qui n'oppose pas rapports sociaux et appartenances ethniques mais qui les distingue et en analyse les interférences. Nous reviendrons plus loin sur l'intégration et la spécificité des situations auxquelles font face les populations musulmanes.

La présente contribution est le fruit d'un travail de recherche à la fois personnel et collectif. En effet, je dirige avec Jean-Yves Causer, depuis plusieurs années déjà, une enquête pédagogique, au sein de la Faculté des sciences sociales de l'Université de Strasbourg, sur le thème des discriminations ethniques (dans un premier temps dans le monde du travail, et depuis 2009 dans la formation et l'enseignement), dans laquelle nous participons de façon active au recueil des données, aux côtés de nombreux étudiants dont je tiens à saluer ici la qualité de l'investissement et du travail, pour la grande majorité d'entre eux. Par ailleurs, j'ai commencé depuis un peu moins de deux ans à mener des entretiens avec des jeunes d'origine maghrébine autour du thème des rapports que l'on peut établir entre la religion, l'identité et l'intégration : ici, le thème de la discrimination apparaît davantage en filigrane, mais il est loin d'être absent.

\section{Esquisse d'une typologie}

Pour rendre compte d'abord de la pluralité des parcours et des opinions et attitudes que l'on constate, pour examiner ensuite les convergences qui se dégagent de ces discours, j'ai construit une typologie ; même si son caractère sommaire et un peu réducteur n'est pas sans poser quelques problèmes, je vais y avoir recours pour rendre mon propos plus clair.

\footnotetext{
4. BERTHELEU, Hélène, "Sens et usages de 'l'ethnicisation'. Le regard majoritaire sur les rapports sociaux ethniques", Revue Européenne des Migrations Internationales, vol. 23, n² 2, 2007, pp. 7-28 (voir p. 11).

5. Ibidem, p. 23.
} 


\section{- La discrimination relativisée}

On repère ainsi un premier groupe, le plus nombreux, dont le discours se caractérise (sur les questions ayant trait à la discrimination) par une ambivalence : les interviewés reconnaissent être ou avoir été confrontés à des attitudes ou des discours discriminatoires à leur égard, ils ressentent les effets d'une vision réductrice et dévalorisante qui les renvoie à une altérité supposée ou réelle et qui a des conséquences néfastes (je pense ici, mais pas seulement, aux rapports avec le monde du travail), mais en même temps ils relativisent ces expériences et mettent davantage l'accent sur des aspects positifs de leurs parcours respectifs.

On trouve dans ce groupe des hommes et des femmes de diverses origines ayant pour point commun, pour la majorité d'entre eux, une trajectoire plutôt marquée par la réussite (notamment scolaire et professionnelle) et par l'intégration dans la société française, même si la diversité est présente pour ce qui concerne des questions telles que la perception de leur identité ou la relation avec la culture d'origine. On peut illustrer cette attitude globale à l'aide des trois extraits d'entretiens.

Pour Farid ${ }^{6}, 21$ ans, célibataire, né en France de parents algériens, titulaire d'un baccalauréat littéraire et ayant quitté l'enseignement su-périeur sans diplôme, travaillant avec un contrat à durée indéterminée (CDI) en tant que vendeur dans une entreprise de téléphonie mobile, il faut relativiser la question de la discrimination: "I Faut pas se poser tout le temps en victime, il n'y a pas plus de racisme en France qu'ailleurs, peut-être moins, mais il faut aller vers les autres et aussi les respecter pour qu'ils te respectent. Ça, mes parents me l'ont appris depuis tout petit et c'est la vérité 11 . Notre interlocuteur semble engagé dans un processus d'intégra-tion qui ne l'empêche pas d'Il être fier " de ses origines et de considérer son appartenance biculturelle comme une richesse.

II en va de même pour Diamila, 24 ans, célibataire, née au Maroc et arrivée en France à l'âge de 2 ans avec sa mère et deux frères pour rejoindre son père; elle a passé un baccalauréat professionnel et travaille comme secrétaire dans une grande entreprise. Selon elle, "I la discrimination, le racisme, ça existe, bien sûr, comme les gens bêtes, mais il ne faut pas exagérer ; il y a surtout des gens qui sont comme moi, tranquilles tant qu'on les agresse pas... Faut voir aussi comment se comportent certains jeunes, qui ne sont pas tous des immigrés d'ailleurs, de vrais délinquants,

6. Les prénoms ont été changés pour respecter l'anonymat auquel nous nous sommes engagés auprès de nos interlocuteurs. 
alors après c'est facile de dire que les gens sont racistes avec eux. Non, je suis en France depuis toute petite et je me sens française. Je sais que je vais rester dans ce pays et que quand je me marierai mes enfants seront français, alors j'essaie de rester moi-même tout en m'adaptant aux coutumes et aux mentalités ici ; ce n'est pas très difficile parce qu'elles me plaisent bien [rires]. Non, évidemment tout n'est pas si facile, mes parents sont contents, mais en même temps ils pensent que je devrais déjà être mariée et mère de famille, et puis surtout ils craignent que i'arrive à la maison avec un fiancé non musulman. Ils ont tort d'avoir peur, mais ça, c'est une autre histoire... "I.

Dans ce second extrait, on voit apparaître (même si l'interviewée est restée superficielle dans le traitement de la question) un sujet qui implique davantage les filles que les garçons, bien que ces derniers soient aussi concernés : la question de la sexualité et du mariage, avec en arrière-plan l'appartenance religieuse. En ce qui concerne les femmes, sont présents à la fois le tabou des relations sexuelles prénuptiales et le désir des parents et de leur entourage de les voir épouser un musulman (si possible de la même origine nationale qu'elles) ; pour ce qui est des hommes, la sexualité avant le mariage suscite moins de résistance, mais on note une certaine crainte de les voir épouser une femme non musulmane, en raison non seulement de l'éloignement de leur communauté, mais aussi parfois par la perception négative de la femme française, moderne, libérée et donc épouse rétive, par conséquent indésirable pour le garçon.

Dans un registre différent, Lucie, 22 ans, célibataire, née d'un père guadeloupéen et d'une mère réunionnaise, ayant suivi une première année universitaire d'espagnol et ayant pour le moment arrêté ses études pour travailler à plein temps comme vendeuse dans un magasin de chaussures à Strasbourg, nous fait également part des expériences de discrimination vécues dans diverses circonstances : "Malgré le fait que je sois française sur les papiers et... et que je le suis [...], parce que j'appartiens à la France... malgré ça, on m'a souvent remis... face à moi, le fait que... du fait que je suis métissée, que je n'étais pas française. Parce qu'un Français, c'est Blanc : voilà [...]. Moi, i'ai eu déjà des réflexions par rapport à ça, parce que quand ça se passait très mal au travail avec mes gérants..., je cumulais mes horaires de travail plus mes horaires de cours, plus les heures de travail perso [...]. Et donc il y a eu un moment où, c'est vrai, i'ai eu une petite baisse de tension, i'étais en plus vraiment très malade, mais bon... Apparemment ce n'était pas une excuse suffisante d'être malade. Donc ma gérante est venue me voir et $m$ 'a dit : "Je ne sais pas si c'est dû à vos origines, mais i'aimerais que vous vous motiviez un peu plus...". J'ai fait : "Pardon ?" ; elle : "Non, mais..." ; i'ai fait : "Non mais, il n'y a rien du 
tout". Voilà, et pourtant Dieu sait que maintenant je la côtoie comme une amie, il n'y a pas de souci. Je sais qu'elle n'est pas raciste, mais c'est pour dire le poids des a priori dans la culture et dans la mentalité des gens 11.

Active, optimiste et entreprenante, notre interlocutrice ne peut pour autant s'empêcher d'exprimer sa préoccupation concernant l'évolution de la discrimination dans la société française. Néanmoins, il y a chez elle, et plus généralement dans ce groupe, une volonté de relativisation des difficultés et des contradictions, qui s'explique d'abord, me semble-t-il, par des parcours scolaires, voire professionnels, plutôt réussis, qui engendrent une confiance à la fois dans sa capacité à élaborer des projets et à surmonter les obstacles, et dans les valeurs de la société qui est désormais la leur.

\section{- La discrimination subie}

Le second groupe a souvent pour dénominateur commun, au contraire du groupe précédent, des difficultés scolaires avec au bout la sortie de l'enseignement avec un bagage scolaire modeste ou une absence de diplômes, et de ce fait une "galère" fréquente en ce qui concerne l'insertion professionnelle. Les filles vont se distinguer davantage des garçons que dans le premier groupe car elles sont assez nombreuses à miser sur un mariage "traditionnel" et un parcours plus semblable à celui de leurs mères, ayant probablement fait le deuil d'une vie professionnelle épanovissante. La discrimination est ici ressentie plus vivement, et il n'est pas rare qu'on lui attribue une responsabilité importante dans les difficultés auxquelles on se trouve confronté. En même temps, l'appartenance à une communauté et/ou à un groupe ethnique est plutôt un élément sécurisant, même si en général le "retour" au pays d'origine ne semble guère à l'ordre du jour ${ }^{7}$. Appuyons-nous également ici sur trois extraits d'entretiens.

Karima, 21 ans, fiancée à un Marocain, née au Maroc et arrivée en France avec sa mère et deux frères à l'âge de 5 ans, sortie du système scolaire après avoir échoué à son BEP et sans emploi au moment de l'entretien, a des idées claires sur la suite qu'elle entend donner à sa vie : " À l'école ça marchait pas très fort, c'était pas mon truc... Alors, ça fait deux ans que je fais des petits boulots par-ci, par-là, i'en ai marre. Et puis, si c'est pour trouver un boulot à l'usine, te faire chier avec les petits

7. Notons au passage que pour la plupart de ces jeunes, tout comme pour ceux des autres catégories analysées, le pays d'origine est plutôt celui des parents car ils sont, eux, nés en France ou arrivés en bas âge. 
chefs racistes et tout ça, non, merci ! L'an prochain, je me marie et puis je reste à la maison; mon fiancé il a un bon travail et il ne tient pas à ce que je travaille, moi. Mon travail ce sera m'occuper de lui et de mes enfants; i'espère en avoir au moins quatre ou cinq, chez nous on est pour les familles nombreuses! [rires]. Non, de toute façon c'est comme ça que i'ai toujours voulu que ça se passe : fonder une famille et me consacrer à elle II. On peut s'interroger sur ses vraies motivations, mais Karima suit l'exemple de sa mère et ne semble pas le faire contrainte et forcée.

Pour les garçons, la revendication de leurs origines et de leur spécificité est, au moins en partie, une façon de se protéger contre la dévalorisation qu'ils ressentent de la part de leur environnement professionnel. On peut prendre pour illustration les propos d'Ahmed, 30 ans, célibataire, né en France de parents algériens, ayant échoué aux épreuves du baccalauréat professionnel et travaillant comme intérimaire dans le secteur de l'électricité industrielle : "T'as beau être français sur ta carte d'identité, la mentalité n'est pas la même, Dieu soit loué, les Français, les vrais, y a que le fric qui les intéresse, le fric et ce qui va avec... On a d'autres valeurs, nous c'est plus la famille et pas "moi, moi, moi !", tu comprends ? [...]. Alors, c'est vrai que i'ai pas beaucoup d'amis français, mais c'est pas facile si t'as pas la même mentalité. Et puis beaucoup sont racistes, même s'ils te font des grands sourires ils te cassent dès que t'as le dos tourné... Bon, au boulot c'est pas grave, chacun est là pour gagner sa vie, pas pour se faire des copains, mais c'est un peu lassant parfois [...]. Rentrer au pays? C'est le bordel là-bas, et même si t'as un travail tu gagnes des clopinettes. Non, je reste ici, et aussi en Allemagne parce que je suis souvent en mission en Allemagne, c'est encore autre chose, je parle pas leur langue mais ils ont leurs qualités, disciplinés, bosseurs... 11.

Discret sur ses projets tant professionnels que personnels, Ahmed donne l'impression de ressentir une certaine amertume du fait d'une inscription dans le monde du travail peu conforme à ses aspirations. II en va quelque peu différemment de Noureddine, 24 ans, célibataire, né en France de parents algériens, ayant obtenu un CAP de couvreur et travaillant en CDI pour une grande entreprise du bâtiment ; dynamique et ouvert, il ne manque pas de projets : "La discrimination? Ben oui, je la rencontre depuis toujours, on te regarde de travers parce que t'as pas la même gueule que tes petits camarades, on te demande tes papiers parce que t'es basané, on te fait pas confiance et on te surveille quand tu rentres dans un magasin... Bon, t'en prends l'habitude, mais parfois ça gonfle! D'un autre côté, en Algérie c'est pas mieux, y a pas de travail, et en plus on te fait sentir que t'es étranger. Alors, c'est ici que je suis chez moi, même si ça fait pas plaisir à 
tout le monde [...]. Dans le boulot ça va, y a des gens avec qui tu t'entends mieux qu'avec d'autres, c'est normal, mais on est là pour gagner sa vie, hein? Moi, bientôt je me marie et je m'installe à mon compte, avec deux potes. Comme ça, t'es ton propre chef et en plus tu peux mieux t'en sortir [...]. Si je me sens algérien ou français? Ni l'un ni l'autre! [clin d'œil et geste à l'appui]. Je me sens moi, c'est tout. Pas Algérien parce que i'ai pas vécu là-bas, i'ai une autre mentalité, plus ouverte. Pas Français parce que on te fait sentir que t'es pas d'ici, pas blanc, pas chrétien, tout ça... Mais tout ça je m'en fous, je vis ma vie, un point c'est tout. Ça m'empêche pas de faire gaffe pour pas choquer mes parents, respecter les traditions ef tout ça, surtout quand je suis à la maison, mais eux c'est eux et moi c'est moi, tu vois? II.

En tout cas, par-delà l'hétérogénéité de ce groupe, la question de la discrimination se pose de façon plus importante et constitue un frein pour l'intégration ou, au moins, une explication pour les difficultés rencontrées.

\section{- La discrimination refoulée}

Le troisième groupe, le plus restreint parmi les personnes rencontrées, concerne celles et ceux qui sont en rupture avec leur famille, mais aussi, bien souvent, avec leur communauté d'origine. Leur rapport à la discrimination est modifié par le fait que, fréquemment, ils ont fait le choix d'un certain renoncement aux valeurs du groupe en question. Cela ne les met pas pour autant à l'abri de la discrimination, mais cette rupture comporte la volonté (plus ou moins consciente) de se fondre dans le groupe majoritaire de la société d'accueil. Dans un certain nombre de cas, il s'agit de femmes pour qui l'émancipation de ce qu'elles ressentaient comme le carcan familial a entraîné cette rupture : tantôt elles refusent de se plier à "la tradition" par le biais d'un mariage imposé, tantôt elles décident de faire leur vie arec quelqu'un que la famille n'accepte pas (souvent un non-musulman), souvent aussi elles rejettent des impositions (permissions de sortie, contrôle de leur vie privée, etc.).

Si pour certaines cette situation est (ou a été) provisoire, avant de retrouver des relations plus ou moins pacifiées avec la famille, pour d'autres, grâce aux bons offices de frères ou de sœurs, voire de leurs mères, cette rupture est souvent vécue comme voulue et définitive. "I'ai tiré un trait dessus II, nous dira, à propos de ses parents Rachida, 29 ans, née en France de parents tunisiens, vivant maritalement, un enfant, titulaire d'un BEP de coiffure, à la recherche d'un emploi. II Ils me traitaient comme une petite gamine : "Où tu vas?, Avec qui ? À quelle heure tu rentres?". J'en 
avais marre, s'ils voulaient que je vive comme eux il aurait fallu qu'ils restent au pays ! [...]. Bien sûr que c'est difficile, il faut se faire sa place ici et il y a des hauts et des bas, mais avec mon mari on se serre les coudes et je veux pas que ma fille vive ce que i'ai vécu "I.

La souffrance est perceptible dans son discours, mais Rachida semble assumer cette rupture et se tourner vers la famille de son compagnon pour la compenser. Pour les hommes, la rupture est parfois le fruit de parcours erratiques et de leur refus d'accepter le jugement parental, mais elle provient aussi dans un certain nombre de cas de choix de vie (concernant notamment la mise en couple) qui les ont conduits à se mettre à l'écart de leur famille. Néanmoins, cette rupture est, pour les uns et pour les autres, toujours source de chagrin, d'autant que ceux et celles qui la vivent se sentent plus ou moins coupables, même s'ils ne le disent en général qu'à demi-mot.

\section{Discrimination et intégration}

En somme, les trois catégories que j'ai créées pour cette typologie (en ayant parfaitement conscience du caractère fragile d'une telle entreprise) se différencient davantage par l'attitude qu'elles ont vis-à-vis de la discrimination que par l'intensité avec laquelle elle les touche, même si on peut penser que les individus se trouvant dans des parcours de réussite côtoient celle-ci moins souvent que ceux qui se trouvent dans des situations plus précaires.

Le premier groupe relativise l'importance de la discrimination; les personnes qui le forment ont plus confiance en leurs capacités pour surmonter les difficultés, y compris celles issues des diverses formes de discrimination, et se sentent aussi plus identifiés avec la société globale. Les membres du second groupe ont davantage tendance à se replier sur la famille et le groupe d'origine pour faire face aux difficultés qu'ils rencontrent, y compris celles liées aux phénomènes discriminatoires; la conformité avec les valeurs traditionnelles est le prix à payer pour ne pas se couper de ce cocon protecteur. Cependant, ce choix s'accompagne de son lot de difficultés et de contradictions dans la mesure où les valeurs et les normes dominantes de la société se sont également, en partie au moins, imprimées en eux ; ainsi, l'individualisme n'est pas absent des récits, y compris chez nos interlocuteurs se présentant comme les plus conformes à "la tradition". Dans le cas du troisième groupe, l'accent est mis plutôt sur les difficultés ressenties au niveau familial (voire communautaire) et sur 
la volonté d'adopter un style de vie "à la française", même si parfois les difficultés rencontrées suscitent révolte ou découragement chez ceux (plutôt nombreux parmi les personnes rencontrées que nous rangeons dans cette catégorie) qui se trouvent en situation de précarité matérielle.

La question de la discrimination des populations d'origine extraeuropéenne nous renvoie immanquablement à celle de l'intégration. D'abord, il faut rappeler ici que l'intégration est un processus dont la composante essentielle est la durée. On peut la rendre plus difficile et lui faire emprunter des parcours chaotiques pouvant aboutir à des échecs ou, au contraire, contribuer à la rendre plus aisée et avec plus de chances de succès, mais que cela implique le blocage ou l'aboutissement rapide du processus semble improbable. Cependant, il n'est pas inutile de comprendre comment ce processus est accompagné par des politiques ad hoc, car il faut insister sur le fait que l'intégration est un phénomène qui n'implique pas seulement les immigrés et leurs enfants, mais également la société du pays d'accueil et la cohésion sociale (le vivre ensemble), et d'abord les pouvoirs publics de la société concernée.

Pour en venir aux facteurs de réussite, il en est un dont le caractère central peut être affirmé : la scolarité des enfants. Or, même si l'école joue globalement un rôle positif et bénéficie d'une bonne image chez la grande majorité des familles immigrées qui ont des enfants scolarisés, des problèmes subsistent. Les processus d'ethnicisation qui se sont développés dans la société française, comme dans bien d'autres, n'épargnent pas l'espace scolaire, et les débats autour de la violence à l'école ou ceux concernant le port du foulard sont des bonnes illustrations d'un climat de défiance à l'égard des "immigrés" (en fait, il s'agit surtout des populations d'origine non européenne). En outre, comme l'écrit Catherine Delcroix, "le phénomène de l'orientation quasi systématique des enfants d'immigré-e-s vers des cursus "technologiques", y compris celles et ceux qui ont de bons résultats scolaires, peut être observé au niveau de maints établissements $1{ }^{8}$. Les situations de discrimination sont, selon toute vraisemblance, moindres à l'école que dans le monde du travail, par exemple, mais elles ont des conséquences très importantes pour les trajectoires des individus concernés. Par ailleurs, l'école, qui intervient très tôt dans les parcours, ne doit pas être vue seulement sous l'angle des apprentissages scolaires, mais aussi en tant que lieu-clé pour la socialisation et l'initiation à la citoyenneté.

8. DELCROIX, Catherine, "Entre volonté de s'en sortir et discrimination, une trajectoire éclairante", Nouvelles Questions Féministes, vol. 26, $\mathrm{n}^{\circ}$ 3, 2007, pp. 82-100 (voir p. 87). 
D'autres éléments importants pour mesurer l'ampleur des difficultés auxquelles se trouvent confrontées les populations que l'on dit "issues de l'immigration" et éventuellement des progrès accomplis dans tel ou tel domaine sont la place que ces populations occupent dans le monde du travail, le logement, la santé, le monde associatif ou encore les questions liées à la représentation politique de ceux (la grande majorité) qui ont la nationalité française. Mais je me contenterai d'aborder une question qui fait l'objet de nombreux débats : les croyances et pratiques religieuses.

Une partie non négligeable des jeunes d'origine maghrébine ou noireafricaine ne sont pas des musulmans pratiquants, voire ne sont pas croyants, semblables en cela à une partie importante des jeunes dits "Français de souche". Cela ne signifie pas qu'ils renient leurs origines ou qu'ils rejettent en bloc une identité que leurs parents leur ont, peu ou prou, transmise, mais ils sont davantage tournés vers la société où ils ont grandi et vers les enjeux auxquels celle-ci les confronte, et ils ont pris leurs distances à l'égard de la religion. D'autres, également nombreux, sont tiraillés par la contradiction qu'ils ressentent entre la fidélité au modèle familial et à l'identité de la société de leurs parents, et l'insertion sociale, scolaire et professionnelle dans la société où ils vivent et dont ils partagent nombre de valeurs et d'aspirations. II ne s'agit pas d'une contradiction entre être "bon musulman" et "bon Français", mais d'une contradiction entre le pays d'origine (qui n'est plus tout à fait le leur) et celui où ils sont souvent nés ou en tout cas celui où ils ont grandi, perçus comme différents et parfois "opposés". Ceux-là sont amenés à "bricoler", avec plus ou moins de bonheur, des compromis. Pour d'autres, enfin, l'idée même de renoncer à un particularisme culturel dont l'élément religieux constitue l'élément central de cohérence est impensable. Cependant, comme l'ont montré nombre d'études, cette identité est fortement marquée par les processus de subjectivation propres aux sociétés modernes, et leur pratique religieuse s'apparente à celle d'autres groupes de croyants de ce type de sociétés. En cela, on peut dire qu'il y a bien, à l'insu même des individus, un "islam à la française" qui se met en place et qui n'est pas celui que leurs parents ont connu'

$\mathrm{Si}$, comme le dit Jocelyne Cesari, "I l'islam fonctionne comme vecteur d'attestation d'une identité collective ", il fournit également " une reconstruction de l'identité à partir du référent islamique qui vient redonner du sens, non seulement individuellement mais aussi collectivement, et favoriser une

9. Voir à ce sujet TIETZE, Nikola, Jeunes musulmans de France et d'Allemagne: les constructions subjectives de l'identité, Paris : Éd. L'Harmattan, 2002, 237 p. 
compréhension du monde environnant. II fonctionne alors comme une "carte", ou une grille de lecture conférant balises et repères dans la conduite individuelle comme dans les interactions sociales $1{ }^{10}$. La religion peut être une boussole que l'individu utilise pour donner du sens aux situations qu'il vit et qui peut l'aider à prendre une part plus active dans une société où il ressent des difficultés pour être pris en compte. Sous cet angle-là, non seulement il n'y a pas de contradiction entre les structures cultuelles et associatives islamiques et une intégration réussie de ceux qui en font partie dans la société globale, mais il y a coïncidence. Toutefois, il ne faut pas pour autant occulter que certaines associations, comme les prédicateurs de certaines mosquées, ont une vision du monde et des sociétés qui les conduit à proposer une grille de lecture qui tend à opposer islam et "intégration". Ceci souligne l'urgence qu'il y a en matière de formation des cadres religieux. En somme, le facteur religieux peut être un élément positif dans le processus d'intégration dans la société comme il peut être un obstacle et même un danger.

\section{Pour conclure}

Les extraits d'entretiens transcris dans la présente contribution illustrent, à leur manière, la diversité de ceux que l'on désigne comme les enfants issus de l'immigration ou la "seconde génération", mais aussi l'ampleur des transformations qui se déroulent dans la société française de la fin du $X X^{e}$ siècle et du début du XXI ${ }^{\mathrm{e}}$. L'immigration est ici une sorte de miroir dans lequel se reflètent les tendances (parfois contradictoires) qui traversent la société française. Pour Jean-Loup Amselle, "I c'est dans les situations de crise sociale, économique, morale, politique ou religieuse que l'étranger comme catégorie assignée s'épanouit particulièrement et il est aisé, de ce point de vue, de mettre en relation les phénomènes d'exacerbation identitaire avec quelques-uns des événements majeurs de notre histoire nationale [...]. La crise que nous vivons actuellement n'est pas tant marquée par le chômage que par l'implosion des frontières nationales, implosion elle-même liée à la construction de l'Europe et à la mondialisation de l'économie. On peut ainsi faire l'hypothèse que la transnationalisation de la politique et de l'économie provoque une crispation identitaire et une modification des référents négatifs. S'il faut toujours un Autre pour se définir soi-même, la nature de cet Autre varie et, dans la conjoncture actuelle, c'est la disparition des

10. CESARI, Jocelyne, "De l'islam en France à l'islam de France", in : DEWITTE, Philippe (sous la direction de), Immigration et intégration : l'état des savoirs, Paris : Éd. La Découverte, 1999, pp. 222-231 (voir p. 230). 
ennemis traditionnels qui entraine l'apparition d'un nationalisme interne [...]. À la lutte des classes qui aurait pu fournir une matrice de remplacement s'est substituée une lutte des nationaux contre les étrangers $1{ }^{11}$.

Bien que toutes ces mutations ne soient pas à proprement parler des crises, l'effacement d'un certain nombre de repères et les incertitudes nées de ces chambardements des mentalités, des modes de vie et de nombre de réalités matérielles conduit une partie des populations autochtones les plus fragilisées, et pas seulement au sein de la classe ouvrière, à désigner un nouveau bouc émissaire responsable des difficultés matérielles, de la frustration, de la peur du lendemain, de tous les malheurs en somme, et ce seront les derniers arrivés qui feront l'affaire. La crise du mouvement ouvrier rendra plus aisée cette opération car, même si celui-ci n'a pas toujours été imperméable au racisme et à la xénophobie, il a plutôt joué un rôle de bouclier contre ceux-ci et d'intégration des populations immigrées dans les entreprises et les quartiers.

Malgré tous les obstacles rencontrés, l'intégration est une réalité à l'œuvre et la diversité des origines sera de plus en plus un signe distinctif de la France du $\left.X X\right|^{e}$ siècle. Cela ne veut pas dire que les problèmes soient résolus, et notamment celui du vivre ensemble. À ceux qui s'opposent à l'intégration au nom de la préservation de je ne sais quelle pureté font écho ceux qui veulent assigner à identité les descendants des migrants au nom de la fidélité au pays d'origine de ceux-ci, à la culture et à la religion d'appartenance, ceux pour qui le multiculturalisme ne se conçoit que comme une superposition de cultures et de groupes "à l'abri de toute contamination"... Les uns et les autres sont animés par les mêmes fantasmes, les mêmes obsessions.

Quant aux partisans d'une intégration assimilationniste, souvent sceptiques quant aux capacités d'intégration des immigrés non européens et de leurs descendants, la préservation de traits identitaires des cultures d'origine n'est perçue que comme résistance à l'intégration. À ce sujet, Alain Touraine nous dit : "I On ne peut pas qualifier de démocratiques les positions libérales qui invitent les immigrés à s'assimiler à une culture et à s'intégrer à une société qui s'identifient elles-mêmes à des valeurs universelles. Passez, leur dit-on, de votre monde fermé à notre monde ouvert. Autant leur demander de se dépouiller de leur culture pour entrer nus dans

11. AMSELLE, Jean-Loup, "Quelques réflexions sur la question des identités collectives en France aujourd'hui", in : FOURIER, Martine ; VERMĖ, Geneviève (sous la direction de), Ethnicisation des rapports sociaux: racismes, nationalismes, ethnicismes et culturalismes (Qu'est-ce que la recherche interculturelle? vol. 3), Paris : Éd. L'Harmattan ; Fontenay - Saint-Cloud : École normale supérieure, 1994, pp. 44-54 (voir p. 53). 
un monde neuf et étranger. Quelle arrogance, quel mépris pour les cultures et les expériences différentes! C'est détruire plus sûrement encore la démocratie que d'accepter un différencialisme absolv et la formation de communautés ayant chacune ses règles. Cet égalitarisme de façade recouvre mal en fait la ségrégation et l'exclusion de minorités considérées comme absolument différentes. C'est, au contraire, aujourd'hui comme hier, en combinant défense de l'identité et intégration qu'on élaborera des solutions démocratiques [...]. L'intégration des immigrants n'est pas réussie quand ils se sont fondus dans la masse ; elle l'est quand les autres respectent leur identité culturelle, parce qu'elle leur apparaît compatible avec l'appartenance à une société commune $11^{12}$.

Entre un assimilationnisme désuet et un communautarisme qui enferme dans une identité "préservée" les individus en fonction de critères ethniques, religieux ou de tout autre nature, c'est une société ouverte, où le multiculturalisme n'est pas une superposition de populations hermétiques les unes aux autres, qu'il nous faudra construire, une société aussi moins axée sur la seule logique du profit et de la compétition.

12. TOURAINE, Alain, Qu'est-ce que la démocratie ?, Paris : Éd. Fayard, 1994, 297 p. 